\title{
Aportes del pensamiento y las ciencias de la Complejidad al estudio de los determinantes de la salud
}

\author{
Contributions of the thinking and sciences of Complexity to \\ the study of health determinants
}

\author{
Rina Milagros Ramis Andalia'; Pedro Luis Sotolongo Codina"' \\ ' Máster en Salud Pública. Epidemióloga. Investigadora Auxiliar. Escuela Nacional de \\ Salud Pública. La Habana, Cuba. \\ "Doctor en Ciencias. Investigador Titular. Academia de Ciencias de Cuba. La \\ Habana, Cuba.
}

\section{RESUMEN}

El desarrollo de las Ciencias de la Modernidad permitió que el saber científico se erigiera como el más importante de todos los saberes, de modo que la "medición" y la "exactitud" se convirtieron en virtudes altamente apreciadas. Sin embargo, descubrimientos científicos realizados durante el siglo xix y xx arribaron a conclusiones opuestas: "la incertidumbre", la "borrosidad" la "emergencia" y "el caos", aparecen como expresiones de una nueva forma de pensar científico que quiebra la cadena de cinco siglos de éxitos de las Ciencias Clásicas. Se plantea la existencia de "vacíos epistemológicos" en la Epidemiología, especialmente relacionados con las categorías epidemiológicas de causalidad y riesgo dado que los problemas actuales de salud se globalizan cada vez más, se tornan "holísticos" y se determinan, no por la acción de factores de riesgo individuales, sino por la participación de "lo social" articulado con "lo biológico" como una totalidad. Se reflexiona acerca de la influencia que ha tenido el pensamiento y las teorías de la Complejidad en la Epidemiología Social Latinoamericana. Se menciona el trabajo que realizan diferentes grupos en Cuba, entre ellos, el Grupo Red de Salud y Complejidad de la Cátedra de Complejidad del Instituto de Filosofía de La Habana y el Grupo de Epidemiología de la Escuela Nacional de Salud Pública, en la búsqueda de alternativas para articular la causalidad y los determinantes desde los presupuestos del pensamiento y las teorías de la Complejidad, de forma que se 
expresen no sólo como metáforas teóricas, sino como tentativa real de aplicación práctica.

Palabras clave: Ciencias de la Complejidad, determinantes de la salud, epidemiología.

\begin{abstract}
The development of Sciences of Modernity made possible that the scientific knowledge arose as the most important of all knowledge in such a way that "measurement" and "exactitude" became highly appreciated virtues. However, the scientific discoveries made during the $x i x$ and $x x$ centuries reached opposing conclusions: "uncertainty", "blurriness", "emergency" and "chaos" appear as expressions of a new way of thinking that breaks the chain of five centuries of successes in Classical Sciences. It is stated the existence of epistemological gaps in Epidemiology, especially in relation to the epidemiological categories of causality and risk, since the current health problems are increasingly globalized, become "holistic", and are determined not by the action of individual risk factors, but by the participation of "the social" articulated with "the biological" as a whole. It is reflected on the influence thinking and the theories of Complexity has had on the Social Latin American Epidemiology. Reference is made to the work done by different groups in Cuba, such as the Health and Complexity Network Group of the Professorship of Complexity of the Institute of Philosophy of Havana and the Epidemiology Group of the National School of Public Health in the search of alternatives to articulate causality and the determinants, starting from the assumptions of thought and the theories of Complexity, in order to express them not only as theoretical metaphors, but also as a real attempt of practical application.
\end{abstract}

Key words: Sciences of Complexity, health determinants, epidemiology.

\title{
I NTRODUCCI ÓN
}

El desarrollo de las Ciencias de la Modernidad había permitido que el saber científico se erigiera como el más importante de todos los saberes, de modo que la "medición" y la "exactitud" que la caracterizaron, se convirtieron en virtudes altamente apreciadas. Sin embargo, descubrimientos científicos realizados durante los siglos xix y xx, arribaron a conclusiones opuestas: "la incertidumbre", la "borrosidad" la "emergencia", "el caos", aparecen como expresiones de una nueva forma de pensar científico que quiebra el ideal de "certidumbre" y "exactitud" reinante tras cinco siglos de indiscutibles éxitos de las Ciencias Clásicas.

Forman parte de esta revolución del saber: los cuestionamientos epistemológicos desde la ciencia, la sociología de la ciencia y el conocimiento. También teorías científicas de nuevo tipo, que se construyen sobre la base de la demolición de los 
ideales de la racionalidad clásica: teoría de catástrofes, teoría del caos, dinámica no lineal: "Complejidad."

La Salud Pública, denominada en sus inicios Higiene Publica, se erige como disciplina científica de alcance social en el siglo xx, teniendo en sus propósitos prestar servicios de salud a la población de forma planificada y organizada, enfrentar plagas y otras enfermedades, evitar la propagación de procesos desfavorables a la salud y de ocurrir, tener capacidad de identificarlos y resolverlos con rapidez, a un mínimo de costos y daños en vidas humanas, orientando a la sociedad hacia la prevención de las enfermedades, la educación y el fomento de formas de vida personal, familiar y social saludables.

Pero ese ideal de Salud Pública fue mutilado a nivel mundial, por la acción de la privatización y la distribución desigual de recursos y riquezas, como consecuencia de la imposición global del sistema capitalista- monopolizador e imperialista que fragmenta y destruye el proyecto humano de desarrollo social. Lograr ese ideal, actualmente implica en primer lugar, luchar contra ese sistema de desigualdades e iniquidades, además de restituir el enfoque holístico, auténticamente poblacional de "lo epidemiológico" (y que incluya lo antropogénico).

A pesar del duro golpe recibido por la comunidad progresista internacional a la caída del campo socialista, otros factores prometedores han emergido en años recientes. Uno de los principales es la creciente capacidad de reacción mundial ante las injusticias y riesgos colectivos. Un nuevo superpoder ha aparecido en escena: "la opinión pública." "2

Otro factor positivo es la toma creciente de conciencia internacional sobre la crisis civilizatoria que se avecina como consecuencia del falso ideal de desarrollo y progreso impuesto por el mundo capitalista, que por un lado ha llevado al agotamiento creciente de las fuentes de recursos naturales como el agua, el aire, combustibles, suelos, alimentos: recursos indivisibles, irremplazables y no reproducibles y por otro, la lucha por el poder hegemónico, mediante el despojo de las economías, el bloqueo, los actos terroristas, y los conflictos bélicos. Se incrementa el enriquecimiento de pocos y la pobreza extrema, crece la injusticia social, las desigualdades e iniquidades, lo que en conjunto amenaza con destruir la humanidad. Este panorama explica las grandes diferencias que se observan en la salud de las poblaciones a nivel mundial, donde los menos favorecidos carecen de recursos y servicios esenciales para garantizar su vida, padecen de desigualdades e iniquidades, que se traducen en mayor número de migraciones, enfermedades, sufrimiento y muerte.

En Cuba, el triunfo de la Revolución permitió a los ciudadanos vivir durante años en una sociedad sin iniquidades sociales, contar con la voluntad política del Estado al distribuir los recursos esenciales con justicia social, crear y poner al alcance de todos servicios de salud y educación gratuita y de elevada calificación, garantizar seguridad social universal, medicamentos esenciales a precios subsidiados por el estado aún en los momentos económicos más difíciles, y disponer de una canasta básica alimentaria para cada individuo desde hace más 49 años. Sin embargo, después de la caída del campo socialista y la intensificación del bloqueo a Cuba por parte de los EE.UU., se hacen evidentes iniquidades que no existían, en medio de una sociedad con apego a la justicia social. Corresponde a los cubanos de ahora, enfrentar estos nuevos desafíos en una etapa de crisis global del modelo civilizador a nivel mundial de resultados y futuro impredecibles, actuar de forma organizada e inteligente para darle continuidad al proyecto revolucionario de garantizar la salud de la población cubana. 
En ese marco, a la salud publica cubana aún le corresponde erradicar completamente el paradigma médico-biologicista perpetuado por el ideal de atención médica curativa que ha privilegiado la práctica profesional de la salud a nivel mundial, incluyendo a Cuba, ya que no obstante los cambios radicales establecidos en la formación integral y el quehacer de los profesionales de la salud, el desplazamiento de las actividades formativas y de los servicios hacia la atención primaria, en la práctica continúa dominando el modelo asistencial, perdiéndose oportunidades de obtener resultados significativos en el área de la promoción y fomento de la salud. Para ello el sector de la salud debe asumir plenamente el paradigma epidemiológico-salubrista y social y actuar intersectorialmente con las instituciones del estado, las organizaciones sociales y los órganos locales de poder. Esto significa, como diría Geoffry Rose, desarrollar estrategias "poblacionales" y no sólo "individuales."

Se trata no sólo de identificar riesgos y desviaciones de la salud, prever, para prevenir o disminuir los efectos indeseables de las enfermedades de forma oportuna es decir, la denominada "tolerancia estrecha o vertical", sino también fomentar la "tolerancia ancha u horizontal" que implica tomar las medidas que permitan resistir lo que adviene, aún sin que se haya podido identificar de antemano; lograr una toma de conciencia con respecto al papel líder del sector de la salud en cuanto a identificar los problemas fundamentales de salud en el territorio. Que el sector de la salud recupere su liderazgo en la comunidad y supere las expectativas alcanzadas en los primeros años de la Revolución donde brilló por su capacidad de convocar a los órganos de gobierno, políticos y de masas en la solución de los problemas de salud a nivel local, que todos los factores de la sociedad perciban que construir la salud de forma holística y transdisciplinar es también una tarea que les corresponde. Una forma de lograr esto es estudiar el proceso salud-enfermedad desde la perspectiva de sus determinantes sociales.

Identificar los determinantes sociales y emprender las estrategias de promoción de la Salud que de ello se deriven, significa elevar la atención de Salud al nivel en que históricamente han estado los servicios de atención a la enfermedad, integrando a todos los actores sociales y las disciplinas científicas para su consecución. Lo anterior redundará en una estrategia mixta o multiplicidad estratégica en la epidemiología; la combinación de una serie de medidas, sabiendo que ninguna dará la solución completa, ni todo el tiempo.

\section{ASPECTOS CONCEPTUALES Y TEÓRI COS DE LO "MI CRO" Y LO "MACRO SOCI AL". LO I NDI VI DUAL Y LO POBLACI ONAL, LA CAUSALI DAD Y LOS DETERMI NANTES}

Virchow revolucionó las ciencias en el siglo xix cuando dijo que las causas de las enfermedades había que buscarlas en la célula, dando por tierra el paradigma de los cuatro humores y sin embargo asumió posiciones mecanicistas frente a la unidad del organismo vivo al querer convertirlo, como dijera Engels: "en una federación de estados celulares", pero también fue pionero al plantear la importancia de los condicionantes sociales y económicos en relación con las causas y consecuencias de esas enfermedades. ${ }^{4,5}$

El problema de la causalidad es uno de los problemas epistemológicos, en los que históricamente se ha debatido la Epidemiología. La enconada lucha de los inicios del siglo xix entre contagionistas y seguidores de la teoría miasmática, aún persistió en el siglo xx y xxi aunque con matices diferentes El abanico de posturas se extiende desde los que proponen generalizar el uso de los postulados clásicos de la causalidad, hasta los que consideran que la Epidemiología debe abandonar el 
concepto de causa y limitarse a dar explicaciones no deterministas de los problemas que investiga. Las críticas al concepto de causalidad fueron formuladas por primera vez por David Hume en 1740 y el asunto aún no ha sido resuelto, por lo que se mantiene en le centro de la polémica. ${ }^{6-8}$

Las propuestas para el estudio de la causalidad durante el pasado siglo $\mathrm{xx}$, se basaron fundamentalmente en los modelos epidemiológicos de causas única/efectos únicos (monocausal) o causas múltiples/efectos múltiples (multicausal). Con el incremento de las enfermedades no transmisibles se hicieron comunes las investigaciones de factores de riesgo, auxiliándose de métodos analíticos y experimentales (el desmembramiento de las totalidades en sus "partes", para analizarlas; con lo que, sin advertirlo, se escapa inevitablemente la Complejidad inherente a esa totalidad), ambos modelos abordaban el objeto de estudio desde una perspectiva clínica o individual. El ambiente y otras causas eran parte de una externalidad no incorporada.

Mas adelante, Susser M, Susser E, desde una visión sistémica pero jerárquica, propusieron el modelo Ecoepidemiológico (cajas chinas), que aunque supera las limitaciones conceptuales del modelo de Factores de Riesgo, ha sido criticado por Breilh de establecer una "dicotomía mecánica entre elementos sociales e individuales relegados a sistemas distintos, negar la relación dialéctica entre fenómenos sociales e individuales, desdeñar al individuo como personificación de hechos sociales fundamentales, su carácter representativo de intereses y relaciones de clases y convertirlo en una unidad funcional influida desde fuera por la clase social que actuaría como algo exterior." 9,10

De modo que utilizando las propuestas metodológicas del modelo de causalidad múltiple de Mc Mahon, ha sido imposible superar los problemas conceptuales que impone la existencia de esta dicotomía y por otra parte, el modelo de los Susser se enfrenta a escollos conceptuales y teóricos que no logra superar.

Se constata pues, un vacío epistemológico en la Epidemiología, especialmente en las categorías fundamentales de causalidad y riesgo ya que los problemas de salud se globalizan cada vez más, se tornan "holísticos" y se determinan cada vez más, no por la acción de factores de riesgo individuales, sino por la participación de "lo social" articulado con "lo biológico" como una totalidad.

La falta de articulación entre lo individual y lo social, ha sido también de índole conceptual, ya que no son iguales las causas que originan la enfermedad en el individuo que en las poblaciones, y se requiere de una teoría científica capaz de articularlas. $^{8}$

En el espacio de lo "micro" funcionan las leyes de lo biológico, de lo individual, cuyas características pueden ser - con cierta frecuencia- contadas y/o verificadas utilizando métodos y técnicas estadísticas, mientras que sus cualidades han de ser indagadas utilizando métodos y técnicas cualitativas. En el espacio de lo "macro social" predominan las regularidades que rigen los procesos sociales, entonces, ¿cómo estudiar el "todo" sin desmembrarlo en sus partes, sin fragmentar el objeto estudiado?

Han sido las ciencias sociales, las que ofreciendo métodos y técnicas flexibles de indagación lograron una mejor aproximación al estudio de "lo social".

Posteriormente, se propuso la triangulación de técnicas con la intención de integrar las diversas formas de investigar en salud lo individual y lo poblacional. 
Articular las causas en ambas dimensiones significa trascender; transitar de lo "micro" a lo "macro social" sin ignorar las regularidades que operan en cada uno de esos espacios, En este sentido y desde diferentes perspectivas y posiciones epistemológicas se han realizado diversas propuestas a través del estudio de los determinantes de la salud, considerados como los causales que rigen el comportamiento del proceso salud-enfermedad en el espacio poblacional. Sin embargo, la aprehensión de "los fenómenos emergentes" continúa siendo, en buena medida, una "asignatura pendiente" para buena parte de la teoría social. $\mathrm{Ni}$ que decir tiene la importancia de dichos fenómenos "emergentes" en la problemática epidemiológica.

El primer modelo teórico que se conoce para el estudio de los determinantes de la salud lo propone Morris en la década del 60 del siglo xx, posteriormente lo hacen Mosley- Chen y Mckewon. Cuando el llamado de Alma-Atá para hacer de la Atención Primaria la estrategia mundial de salud, surge en 1974 el primer modelo canadiense de Laframboise-Lalonde, seguido de Dever y Blum, en EE.UU. y más adelante las nuevas propuestas de Evans, Stoddart, Marmot, Frank, Wilkinson, Raphael y otros. ${ }^{11,12}$

Mención especial merecen los Latinoamericanos Asa Cristina Laurell de México, Jaime Breilh de Ecuador, Naomar Almeida da Filho, Brazil, Pedro Luis Castellanos de Venezuela, cuyas propuestas se nutren de las categorías "clase social", "carga de trabajo", "modo de vida", "condiciones de vida" "perfil de salud-enfermedad", desde una perspectiva filosófica materialista-dialéctica. ${ }^{13-16}$ (Castellanos PL. Epidemiología, Salud Publica, Situación de Salud y Condiciones de Vida: Consideraciones conceptuales. Seminario Latinoamericano sobre condiciones de vida y salud. 13 de Diciembre de 1995).

Desde los años 20 del pasado siglo, el Ministerio de Sanidad de la antiguo Estado Socialista Soviético (URSS), desde una postura filosófica marxista-leninista, identificó que para la Higiene Social Marxista, el modo y las condiciones de vida eran los determinantes esenciales del estado de salud de la población. A partir de ello se creó la disciplina de Higiene Social y el Instituto de Higiene Social marxista. ${ }^{5}$

En Cuba, Aldereguía en el año 1986, propuso un modelo denominado HombreNaturaleza-Sociedad que consideraba al modo y a las condiciones de vida, los determinantes fundamentales del estado de salud de la población e inmediatamente incluía a la salud pública, el medio ambiente y la biología humana, partiendo de una formación económico-social determinada y teniendo en cuenta los avances de la Revolución Científico-Técnica. ${ }^{5,12}$

Muy recientemente en el Instituto de Higiene Epidemiología y Microbiología, se han iniciado una serie de investigaciones para identificar los determinantes del estado de salud desde la realidad de la población cubana, a partir de las propuestas metodológica de la OMS. ${ }^{11}$

Desde una perspectiva holística, Nancy Krieger, conocida investigadora de la Universidad de Harvard, indaga las causas de los patrones de enfermedad entre niveles, vías y fuerzas y su articulación, argumentando su nueva teoría ecosocial. Se pronuncia a favor de desterrar para siempre de la salud pública el término de "lo próximo" (upstream) y lo "distal" (downstream) argumentando que "lo social" subyace en el dominio de lo distal y lo biológico. La autora identifica "patrones sociales de enfermedad" expresando que "tales patrones sociales de enfermedad representan las consecuencias biológicas de la forma diferenciada de vivir y 
trabajar permitidas a cada grupo social producidas por la sociedad, su economía y prioridades políticas." ${ }^{17}$

La mayoría de las propuestas metodológicas conocidas, tanto para el estudio de los modelos causales como para el estudio de los determinantes de la salud adolecen de la imposibilidad de las Ciencias Clásicas de estudiar el todo sin fragmentarlo en sus partes. Algunas propuestas basadas en teoría de sistemas, o bien no superan la concepción de sistemas lineales o si tienen en cuenta la no linealidad de los sistemas incluyen niveles de jerarquía entre ellos, en detrimento excesivo de las circunstancias no jerárquicas (distribuidas) de diversos ámbitos sociales. Las propuestas de Susser, Bergonzolli, Castellanos son un ejemplo de ello y en la más reciente, emitida por Nancy Krieger, no se detalla como se articulan sistémicamente los niveles, las vías, las fuerzas y los patrones sociales de enfermedad. ${ }^{12,17}$

Almeida Filho, refiriéndose al modelo de Krieger, lo considera inspirado desde una perspectiva fractal, donde el elemento de fractalidad -la autosimilaridad transescalar- sería justamente la interpenetración entre lo biológico y lo social, repetida en todos los niveles. El propio autor comenta que "infelizmente la autora no presenta ejemplos o tentativas de aplicación, avanzando poco allende la formulación preliminar de esta atrayente proposición, conscientemente planteada como una metáfora teórica." ${ }^{18}$

Desde el pensamiento de la Complejidad, Pedro Luis Sotolongo Codina, fundador de la Cátedra de Complejidad del Instituto de Filosofía de La Habana, ha presentado su teoría de práctica social y vida cotidiana, basada en patrones de interacción social con capacidad para articular "lo micro" y "lo macro social" que puede servir de utilidad para la comprensión de estos procesos. ${ }^{19}$

\section{¿QUÉ APORTAN EL PENSAMI ENTO Y LAS CI ENCI AS DE LA COMPLEJIDAD?}

Descubrimientos científicos relacionados con las ciencias de la Complejidad se vienen realizando desde el pasado siglo, muchos de sus antecedentes se encuentran en las ciencias naturales, por ejemplo "el credo determinista de Laplace" llegó a su fin en 1927 con Werner Karl Heisenberg (1901-1976) físico y Premio Nobel alemán, que desarrolló un sistema de mecánica cuántica y cuya indeterminación o principio de incertidumbre ha ejercido una profunda influencia en la física y en la filosofía del siglo xx .1,20,21

Un demoledor y definitivo golpe al "credo" de que sólo a través de las leyes probabilísticas es posible obtener conocimiento científico "causal" y al postulado de que a grandes causas corresponden siempre grandes efectos y viceversa lo aportó un descubrimiento "casual" realizado en 1961 por el meteorólogo del Massachussets Institut of Technology (MIT), Edward Lorenz, quién , un año antes había realizado predicciones probabilísticas del tiempo con 12 ecuaciones y 6 cifras decimales y posteriormente sólo utilizó 3 cifras decimales, quedó sorprendido de las diferencias en los pronósticos. Así surge la teoría del caos. Al efecto que tienen las pequeñas diferencias iniciales se les dio el nombre de "efecto mariposa."

Lorenz intentó encontrar un sistema menos complejo que dependiera sensitivamente de las condiciones iniciales, simplificó el sistema de ecuaciones, se quedó con tres ecuaciones y finalmente observó que los resultados seguían dependiendo de las condiciones iniciales, un comportamiento aparentemente hecho al azar, sin embargo, al verlos en un gráfico, la salida quedó en un espiral doble, un número 8 invertido, como las "alas de una mariposa"....Las ecuaciones de 
Lorenz son definitivamente ordenadas, nunca se pararon en un punto, ni se repitieron, o sea, no son periódicas.

La geometría fractal ha realizado aportes fundamentales a la teoría de la Complejidad, quizás la característica más citada, incluso por el propio formulador de la teoría, el ingeniero francés Benoit Mandelbrot (1975), sea la de que un objeto fractal puede ser subdividido reiteradamente, hasta el infinito, presentando en cada una de estas iteraciones una semejanza con el conjunto. Una representación gráfica de este fenómeno está en las ramificaciones o arborescencias, tan típicas, del sistema pulmonar, nervioso o sanguíneo del cuerpo humano, en el que cada parte se asemeja al todo.

El análisis fractal pone de manifiesto qué y cómo la constancia genera innovación, qué y cómo lo idéntico es distinto, o en otras palabras, qué y cómo lo cuantitativo puede adquirir trascendencia cualitativa. Un ejemplo de esto se puede ver en la variable cuantitativa "tamaño del grupo", la cual tiene trascendencia cualitativa. En efecto, dentro de ciertos límites, al aumentar el número de miembros, varían esencialmente el estilo de vida y los problemas del grupo hasta el extremo de que se puede afirmar en determinados supuestos que se está ante un grupo nuevo.

En general, los fenómenos psicológicos y sociales tienen propiedades fractales: la conducta imitativa, los procesos de enculturación y de socialización, la organización formal de las empresas, la transmisión de rumores y otros.

Otra teoría que fundamenta la ciencia de la Complejidad es la denominada "Teoría de los conjuntos Borrosos". Desde la década de los sesenta, y sin entrar en los antecedentes, Lofti A. Zadeh (1965), un ingeniero iraní que trabaja en Berkeley, viene elaborando una teoría de los conjuntos borrosos (fuzzy sets), que trata de formalizar en un modelo lógico y matemático lo impreciso, lo difuminado, lo indeterminado, lo difuso.

Un conjunto borroso no cumple los principios aristotélicos de contradicción y de tercero excluido. Esto significa que una cosa puede pertenecer y no pertenecer a la vez a un mismo conjunto, simplemente porque los criterios de pertenencia no son nítidos.

A partir de ahí, las operaciones lógicas no responden a la estadística de la probabilidad ni por tanto a la frecuencia de un fenómeno, sino que construyen el razonamiento en términos de posibilidad, que son cualitativos y se refieren a las capacidades y virtualidades.

El tema de la continuidad y la discontinuidad, que late en la lógica difusa, está también presente en otra teoría, que se mueve en un ámbito muy diferente a aquélla, la llamada "Teoría de las Catástrofes."

A comienzos de los años setenta, el matemático René Thom (1972) presentaba una teoría de la morfogénesis y la estabilidad estructural, conocida poco después como "Teoría de las Catástrofes", nombre que si bien tiene connotación que parece haber contribuido al interés por esta teoría también es fuente de malentendido porque su denotación levanta falsas expectativas acerca del objeto que trata.

Sobre una base topológica pero también filosófica, esta teoría describe los cambios "repentinos"que ocurren en un sistema sin perjuicio de su estabilidad o continuidad; expresado con otras palabras, que el sistema consigue mantenerse gracias a una maniobra de subsistencia. 
Christopher Zeeman (1977) ha hecho de esta teoría, que en principio es descriptiva, una teoría predictiva y en este sentido la ha aplicado a la comprensión de comportamientos sociales, tanto animales como humanos, desde la agresión en el perro, los motines en las cárceles y las reacciones de la bolsa de valores, hasta los conflictos internacionales.

A nivel psicosocial, la teoría de las catástrofes - o bifurcaciones- podría contribuir a un esclarecimiento de procesos como la toma de decisiones o los cambios bruscos de opinión. Y parece especialmente aplicable al proceso de socialización, entendido este como una sucesión de crisis cuyas alternativas van desarrollando socialmente al sujeto, esto es, forman su personalidad en el doble aspecto individual y social. ${ }^{1,20,21}$

El enfoque de la Complejidad desde la perspectiva de los Determinantes sociales no es algo reciente, la Epidemiología y la Medicina Social latinoamericana lo adoptaron desde la década del 80. En la propuesta de Pedro Luis Castellanos de enfrentar el estudio de los determinantes según condiciones de vida, lo etnográfico de Naomar Almeida, el enfoque de Epidemiología Crítica y Contrahegemónica de Jaime Breilh, se identifica el enfoque holístico, emergente, contextualizado y transdisciplinar que caracteriza al "pensamiento complejo."10, 16,18 (Castellanos PL. Epidemiología, Salud Pública, Situación de Salud y Condiciones de Vida: Consideraciones conceptuales. Seminario Latinoamericano sobre condiciones de vida y salud. 13 de Diciembre de 1995).

Entre las escuelas norteamericanas, caracterizadas por su apego al pensamiento analítico propio de las ciencias clásicas, se diferencia el planteamiento de Investigadores de la Escuela de Salud Publica de la Universidad de Harvard, donde figuras como Richard Levins, Tamara Awerbuch y otros que pertenecientes al departamento de la Salud Pública Internacional, se han aproximado durante años al pensamiento y teorías de la Complejidad. Procedente de esa Universidad, se distingue también la ya mencionada investigadora Nancy Krieger autora de la teoría ecosocial. ${ }^{17}$

\section{DESARROLLOS RECI ENTES EN CUBA}

En Cuba desde finales de la década del 80 del pasado siglo, la Escuela de Salud Pública, en su antigua ubicación en el Instituto de Ciencias Médicas y Preclínicas "Victoria de Girón" comenzó a enseñar dos modelos para el estudio de los determinantes de la salud, uno basado en el modelo propuesto para Canadá por Marc Lalonde y otro propuesto por la OPS y que respondía al modelo de condiciones de vida de Pedro Luis Castellanos. En 1998 se brindó el primer curso de posgrado de "teoría de la Complejidad" impartido por Rafael Araujo, promovido por Silvia Martínez Calvo y recibido por un grupo de profesores de la antigua escuela, incluyendo a la autora de este artículo.

Desde el pensamiento de la Complejidad, Pedro Luis Sotolongo Codina, fundador de la Cátedra de Complejidad del Instituto de Filosofía, ha presentado su teoría de práctica social y vida cotidiana, basada en patrones de interacción social con capacidad para articular "lo micro" y "lo macro social."19

En el reciente libro de este investigador cubano, Teoría Social y Vida Cotidiana: La Sociedad como un Sistema Dinámico Complejo, muestra argumentadamente dicha circunstancia, así como la fractalidad de los "patrones de interacción social" (las pautas o regímenes de prácticas colectivas características de la vida cotidiana). 
Los elementos básicos de este paradigma pueden ser descritos resumidamente en los siguientes aspectos:

1. El cambio en la noción misma de Complejidad. En el sentido clásico, la medida de la Complejidad está dada por el grado de dificultad para la comprensión, la complicación de los aparatos matemáticos (los sistemas de cálculo y ecuaciones) empleados, y se considera lo complejo como un atributo indeseable de la realidad, en gran medida producto de la incapacidad para expresarla mejor. En todo caso, un atributo reducible, posible de ser expresado y entendido mediante sistemas más o menos complicados de formulaciones más simples, sean estas verbales o la expresión matemática a través de un sistema de ecuaciones lineales. La nueva noción de lo complejo lo entiende como atributo irreductible de la naturaleza. "Un atributo ordinario y cotidiano que no se había tomado en consideración antes."

"Lo complejo se manifiesta sobre todo en que los sistemas de la naturaleza no sólo no son dados de antemano, sino que devienen en el transcurso mismo de la interacción. Las propiedades del mundo y sus objetos son emergentes. Esto es de suma importancia para la consideración del universo social, donde la emergencia es un atributo característico permanente."

2. La naturaleza tiene un carácter sistémico, integrador, no reducible al campo de ninguna disciplina científica especial. Desde el punto de vista metodológico el holismo tiene preeminencia sobre el reduccionismo. Para la distinción de lo social es pertinente tomar en consideración este aspecto metodológico, pues la consideración de la integralidad de lo social implica aceptar que los enfoques científicos separados y desligados uno de otro son incompetentes para juzgar y comprender la naturaleza de los fenómenos que se investigan.

3. Las relaciones de determinación se caracterizan por la emergencia del orden a partir del desorden, y la superposición del "caos" y el "anti-caos". En el conocimiento del orden del mundo son tanto o más importantes los patrones que se configuran en el devenir de los sistemas, que las determinaciones rígidas. La predicción es posible, pero dentro de los marcos de indeterminación que el propio sistema porta al ser entidad no hecha, devenir (Delgado Díaz CJ. Hacia un nuevo saber: Problemas del enriquecimiento moral del conocimiento humano. Palacio de las Convenciones. La Habana, 8 al 11 del 2002).

\section{¿QUÉ APORTA EL PENSAMIENTO Y LAS TEORÍ AS DE LA COMPLEJ IDAD AL ESTUDIO DE LOS DETERMI NANTES DE LA SALUD?}

Aporta principalmente una nueva forma de pensar en las Ciencias, de modo que:

1. El proceso salud-enfermedad se estudia como sistema complejo que se caracteriza por ser abierto, sujeto a dinámicas no lineales, no jerárquicas e intercambiar sustancias (masa), energía, información y sentido con el entorno.

2. En estos sistemas no rigen las leyes de los pequeños ni de los grandes números, de modo que no son reducibles a funciones determinísticas de la matemática clásica ni estadísticas probabilísticas.

3. En los procesos que se valoran bajo esta nueva conceptualización, las causas y los efectos dependen de las condiciones iniciales en que interactúen en un contexto específico. No existe proporción directa entre causa y efecto, de modo que pequeñas causas pueden determinar grandes consecuencias o viceversa. 
4. Todo proceso de valoración de la causalidad y la determinación de la salud que se estudie a través del pensamiento y las teorías de la Complejidad posee las características de no ser reducible a alguno de sus componentes, ser impredecible, pero previsible y prevenible.

5. La temporalidad en los procesos complejos podrá ser valorada en una doble dimensión, una temporalidad interna (endógena a la totalidad compleja, dada por el transcurrir mismo de sus interacciones internas no lineales) para los diferentes momentos en que se suceden los acontecimientos en el interior de cada uno de los componentes y una temporalidad externa para relacionar el tiempo que transcurre en el espacio contextualizado entre los diversos componentes que interactúan.

6. Los sistemas que se estudian desde la mirada de la Complejidad, tiene carácter auto-organizante y "emergente", esto significa que van conformándose espontáneamente desde sus formas más simples hasta lograr, en ese devenir, alcanzar estadios o manifestaciones superiores dentro de su contexto, ya que a su vez están sujetos a constreñimientos permanentes del entorno (y de sus propios componentes tramados en red) en el cual se desenvuelven. Se distingue por tanto, emergencia, morfogénesis, transformación, es decir, cambio.

7. Los sistemas complejos, evolucionan desde las formas de comportamiento estables a inestables y viceversa, retroceden, avanzan, se superan y se transforman para aparecer bajo formas diferentes. Otras de las características de los sistemas complejos, es la reflectividad, es decir, la capacidad de articularse sobre sí mismo, de modo que cada vuelta, bucle o ciclo se erige en entrada o condición de partida del siguiente (Sotolongo Codina PL. Summary of main ideas at the 1-St Havana Complexity Seminar, January 7th-11 th. Presentado en el Taller "Complejidad y Filosofía". Boston, 2002).

\section{CONSI DERACI ONES FI NALES}

El enfoque de la causalidad y de los determinantes de la salud continúa siendo objeto de debate a nivel internacional, teniendo en cuenta dos posicionamientos epistemológicos fundamentales: por un lado, el paradigma analítico de las Ciencias Clásicas, que se expresa por la acción de una causalidad lineal tanto en el modelo unicausal como multicausal, y que corresponde al ámbito de "lo individual" , donde rigen las leyes de las estadísticas probabilísticas ampliamente tratado por la Epidemiología y las Ciencias Clásicas y por otro, el paradigma holístico de la Complejidad, donde el objeto de estudio es la población como un todo indivisible, la causalidad que actúa no es lineal y el modelo de indagación acude a las Ciencias Sociales para explicar las causas de las causas: los Determinantes Sociales.

Este último modelo no permite el análisis fraccionado del objeto de estudio, se basa en la dinámica de interacciones complejas que se dan en el contexto, donde lo social está continuamente mediando, articulando los procesos y debatiéndose entre emergencias (desde abajo) y constreñimientos (desde arriba).

El estudio de los Determinantes Sociales promovido a escala mundial por la OMS, lleva en América Latina la impronta del pensamiento complejo insertado en la Epidemiología Social y la Medicina Social latinoamericana de los últimos años. 
Los desarrollos científicos de la Complejidad comienzan a ser recientemente explorados. Es de esperar que se perfeccionen y surjan nuevos abordajes para el estudio de los determinantes sociales. Aún no existe consenso sobre como se articulan las causas del proceso salud-enfermedad en el individuo con las determinantes de la salud en la población, aunque se cuenta con varias propuestas. $^{5}$, 9, 11, 12, 15-19 (Castellanos PL. Epidemiología, Salud Publica, Situación de Salud y Condiciones de Vida: Consideraciones conceptuales. Seminario Latinoamericano sobre condiciones de vida y salud. 13 de Diciembre de 1995).

Desde la directiva del grupo Red de Salud y Complejidad del Instituto de Filosofía, apoyado por el Grupo de Epidemiología de la Escuela Nacional de Salud Pública (ENSAP), en colaboración con el Centro de Complejidad y Salud de Camagüey y el Centro de Matemáticas aplicada a la Medicina (CECAM), se estudian alternativas para articular la causalidad y los determinantes sociales basados en los presupuestos del pensamiento y las teorías de la Complejidad con el ánimo de expresarlos no sólo en un contexto teórico, sino también, como tentativas reales de aplicación práctica.

\section{REFERENCI AS BI BLI OGRÁFI CAS}

1. Sotolongo Codina PL. La incidencia en el saber social de una epistemología de la Complejidad contextualizada [Monografía en CD ROM. Ed. Genesis Multimedia]. La Habana: Instituto de Filosofía CITMA; 2002.

2. Berlinguer G. Determinantes sociales de las enfermedades Rev Cubana Salud Pública. 2007; 33 (1).

3. Rose G. Individuos enfermos y poblaciones enfermas. En: OPS. El desafío de la Epidemiología. Problemas y lecturas seleccionadas. Washinthon, D.C.: OPS; 1989. (Publicación Científica; 505).

4. Abeyá Gilardón E. Cartas al Editor. Arch Argent Pediatr. 2008; 106(1): 92-4.

5. Aldereguía Henríquez J. Problemas de Higiene Social y Organización de la Salud Pública. La Habana: Editorial Científico Técnica; 1985

6. Martínez Navarro JF. La Epidemiología en el Pensamiento Científico. Rev San Hig Púb. 1992;66(5-6): 245-50.

7. Silva Ayçaguer LC. Hacia una cultura Epidemiológica Revitalizada. Revista HUMANA. 1997; 1(5):23-33.

8. López Moreno S. Acerca del Estatuto Científico de la Epidemiología. Salud Pública México. 1998; 40(5): 389-91.

9. Susser M, Susser E. Choosing a future of Epidemiology. Part II. From black box to Chinese boxes and ecoepidemiology. Am J Public Health. 1996;86(5):674-7.

10. Breilh J. Epidemiología: Economía, Medicina y Política. Ecuador: SESPAS; 1980: 113-7. 
11. Álvarez Pérez AG, García Fariñas A, Bonet Gorbea M. Pautas conceptuales y metodológicas para explicar los determinantes de los niveles de salud. Caso Cuba. La Habana: INHEM; 2005.

12. Ramis Andalia R. Estado de salud de la población [sitio en Internet]. 2008 [ citada 2008 jun 20]. Disponible en :

http://uvirtual2.sld.cu/wssc/slides/slides. php3?c=63\&d=33\&sz=small

13. Granda E. ¿A qué llamamos salud colectiva, hoy? Rev Cubana Salud Pública. $2004 ; 30(2)$.

14. Granda E. Algunas reflexiones a los 24 años de ALAMES. Medicina Social. $2008 ; 3(2)$.

15. Breilh J. Epidemiología crítica. Ciencia emancipadora e interculturalidad. Buenos Aires, Argentina: Buenos Aires; 2003.

16. Iriart C, Waitzkin H, Breilh J, Estrada A, Merhy E. Medicina social latinoamericana: aportes y desafíos. Rev Panam Salud Pública. 2002;12 (2): 12836.

17. Krieger N. Proximal, distal and politics of causation: What's levels got to do with it? Am J Public Health. 2008;98(2):221-30.

18. Almeida da Filho N. Complejidad y transdisciplinariedad en el campo de la salud colectiva: Evaluación de conceptos y aplicaciones. Salud colectiva.

2006; 2(002): 123-46.

19. Sotolongo Codina PI. Teoria Social y Vida Cotidiana: La sociedad como sistema dinámico complejo. La Habana: Acuario; 2006.

20. Munné F. Universidad de Barcelona. Las teorías de la Complejidad y sus implicaciones en las ciencias del comportamiento. Rev Interam Psicol.

1995; 29(1): 1-12.

21. Ramis Andalia RM. La causalidad compleja: ¿un nuevo paradigma causal en Epidemiología? Rev Cubana Salud Pública. 2004;30(3).

Recibido: 28 de agosto de 2008.

Aprobado: 24 de octubre de 2008.

Rina Milagros Ramis Andalia. Escuela Nacional de Salud Pública. Calle Línea esq, a I. El Vedado 10400. La Habana, Cuba.

E-mail: rina.ramis@infomed.sld.cu, pedro.sotolongo@infomed.sld.cu 